University of Wollongong

Research Online

Faculty of Engineering and Information

Faculty of Engineering and Information

Sciences - Papers: Part B

Sciences

2017

\title{
Accurate range estimation for an electric vehicle including changing environmental conditions and traction system efficiency
}

Kaveh Sarrafan

University of Wollongong, ks282@uowmail.edu.au

Danny Sutanto

University of Wollongong, soetanto@uow.edu.au

Kashem M. Muttaqi

University of Wollongong, kashem@uow.edu.au

Graham Town

Macquarie University

Follow this and additional works at: https://ro.uow.edu.au/eispapers1

Part of the Engineering Commons, and the Science and Technology Studies Commons

Research Online is the open access institutional repository for the University of Wollongong. For further information contact the UOW Library: research-pubs@uow.edu.au 


\title{
Accurate range estimation for an electric vehicle including changing environmental conditions and traction system efficiency
}

\begin{abstract}
Range anxiety is an obstacle to the acceptance of electric vehicles (EVs), caused by drivers' uncertainty regarding their vehicle's state of charge $(\mathrm{SoC})$ and the energy required to reach their destination. Most estimation methods for these variables use simplified models with many assumptions that can result in significant error, particularly if dynamic and environmental conditions are not considered. For example, the combined efficiency of the inverter drive and electric motor varies throughout the route and is not constant as assumed in most range estimation methods. This study proposes an improved method for SoC and range estimation by taking into account location-dependent environmental conditions and timevarying drive system losses. To validate the method, an EV was driven along a selected route and the measured EV battery SoC at the destination was compared with that predicted by the algorithm. The results demonstrated excellent accuracy in the $\mathrm{SoC}$ and range estimation, which should help alleviate range anxiety.
\end{abstract}

\section{Keywords}

system, range, efficiency, changing, accurate, including, environmental, vehicle, conditions, electric, traction, estimation

\section{Disciplines}

Engineering | Science and Technology Studies

\section{Publication Details}

K. Sarrafan, D. Sutanto, K. M. Muttaqi \& G. Town, "Accurate range estimation for an electric vehicle including changing environmental conditions and traction system efficiency," IET Electrical Systems in Transportation, vol. 7, (2) pp. 117-124, 2017. 


\title{
Accurate Range Estimation for an Electric Vehicle Including Changing Environmental Conditions and Traction System Efficiency
}

\author{
Kaveh Sarrafan $^{1 *}$, Danny Sutanto ${ }^{1}$, Kashem M. Muttaqi $^{1}$, Graham Town $^{2}$ \\ ${ }^{1}$ Department of Electrical, Computer and Telecommunications Engineering, University of \\ Wollongong (UoW), Wollongong, NSW 2522, Australia. \\ ${ }^{2}$ Department of Engineering, Macquarie University, NSW 2109, Australia \\ ks282@uowmail.edu.au
}

\begin{abstract}
Range anxiety is an obstacle to the acceptance of electric vehicles (EVs), caused by driver uncertainty regarding their vehicle's state of charge (SoC) and the energy required to reach their destination. Most estimation methods for these variables use simplified models with many assumptions that can result in significant error, particularly if dynamic and environmental conditions are not considered. For example, the combined efficiency of the inverter drive and electric motor varies throughout the route and is not constant as assumed in most range estimation methods. This paper proposes an improved method for SoC and range estimation by taking into account location-dependent environmental conditions and time-varying drive system losses. To validate the method, an EV was driven along a selected route and the measured EV battery SoC at the destination was compared with that predicted by the algorithm. The results demonstrated excellent accuracy in the SoC and range estimation, which should help alleviate range anxiety.
\end{abstract}

\section{Introduction}

EVs are one of the most promising technologies for providing energy security and pollution reduction. One of the main obstacles for the acceptance of EVs is the range anxiety. Range anxiety is a driver's fear of being stranded by a depleted EV battery. From a buyer's perspective, the uncertainty of the amount of remaining battery capacity to reach the destination remains an uneasy thought [1]. Increased uptake of EV subsystems will be facilitated by accurate estimation of the remaining EV battery SoC and the vehicle's range relative to the destination. To achieve this, accurate SoC and range estimation methods need to be developed to provide an accurate estimation of the amount of energy available from the battery at each instant in time. The improved methods should take into account location and time-dependent variables, including the varying efficiency of each component of the EV and the varying environmental conditions and driver behaviours during the planned travel.

In most reports many assumptions were made in the energy estimations and datasheets, or simple theoretical models of the traction motor for losses and efficiency calculation were used [2,3,4,5]. For example, Vaz et al [5] assumed that the road is flat without any slopes, stop signs, or traffic congestion, resulting in overestimated range. Shankar et al [3] reported on a framework for energy consumption 
prediction of EVs over a route under real-world driving conditions (considering traffic type and congestion). The accuracy of the prediction of the proposed method varied between $20-30 \%$ and $70-80 \%$ [3]. Prins et al [4] disregarded the regenerative braking system for energy estimation model in EVs and used a simple vehicle efficiency data which is predicted by the motor manufacturer. Prins [4] claimed that only an $8 \%$ difference exists between the total measured required energy, compared to the predicted values.

Tannahill et al [1] reported means of reducing range anxiety due to EV by taking into account a variety of environmental and behavioural factors. The results show that the predicted result is much closer to the measurement result compared to those using simplified models. However, [1] makes use of the data from the motor manufacturer to determine the losses and efficiency for the motor and motor drive converter, rather than calculating it using the dynamic model of the motor. Further, [1] assumes that these values are constant throughout the driving period.

In this paper, it is proposed to improve the work for SoC and range estimation reported in [1] by taking into account location-dependent environmental conditions and time-varying drive system losses. The results from a validation drive have demonstrated the great accuracy in the SoC and range estimation.

\section{Energy Estimation of EV over a Given Route}

Most reported papers used manufacturer's data sheets for evaluating the efficiency of the motor and the motor drive converter $[1,2,3]$ and they are assumed to be constant throughout the route. This might not be a valid assumption, particularly when dynamically changing environmental and behavioural factors are considered. A more accurate estimation of these values needs to be evaluated in the time domain simulation of the drive system and this requires an understanding of the EV architecture to evaluate the energy usage of each component of the architecture.

A simple model of the EV architecture has four main elements: Energy storage system, transmission system, electric motor and power electronics converter [6]. The energy storage unit can be charged from the grid and also through the regenerative braking system. To accurately evaluate the SoC and hence the remaining distance to destination, the amount of the energy required from the battery at every instance of time needs to be evaluated. The required energy can be calculated from traction (wheel) power, the transmission gear ratio and efficiency, and the losses in the drive system. The effect of traffic and accessory power also need to be taken into account.

An outline of the procedures to estimate the energy and power drawn from the battery at each instant of time, based on the EV architecture in Fig. 1 during the driving duration, taking into account the environmental conditions and efficiency modelling is shown in Fig. 2. 


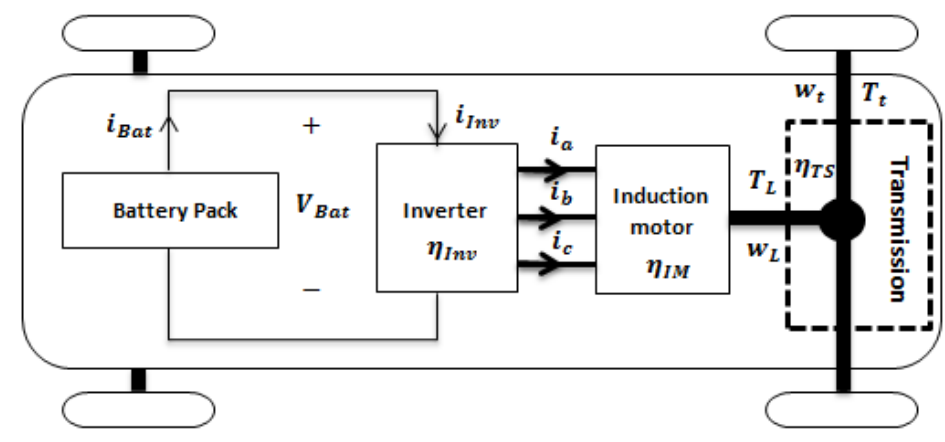

Fig. 1. Schematic view of a simple EV

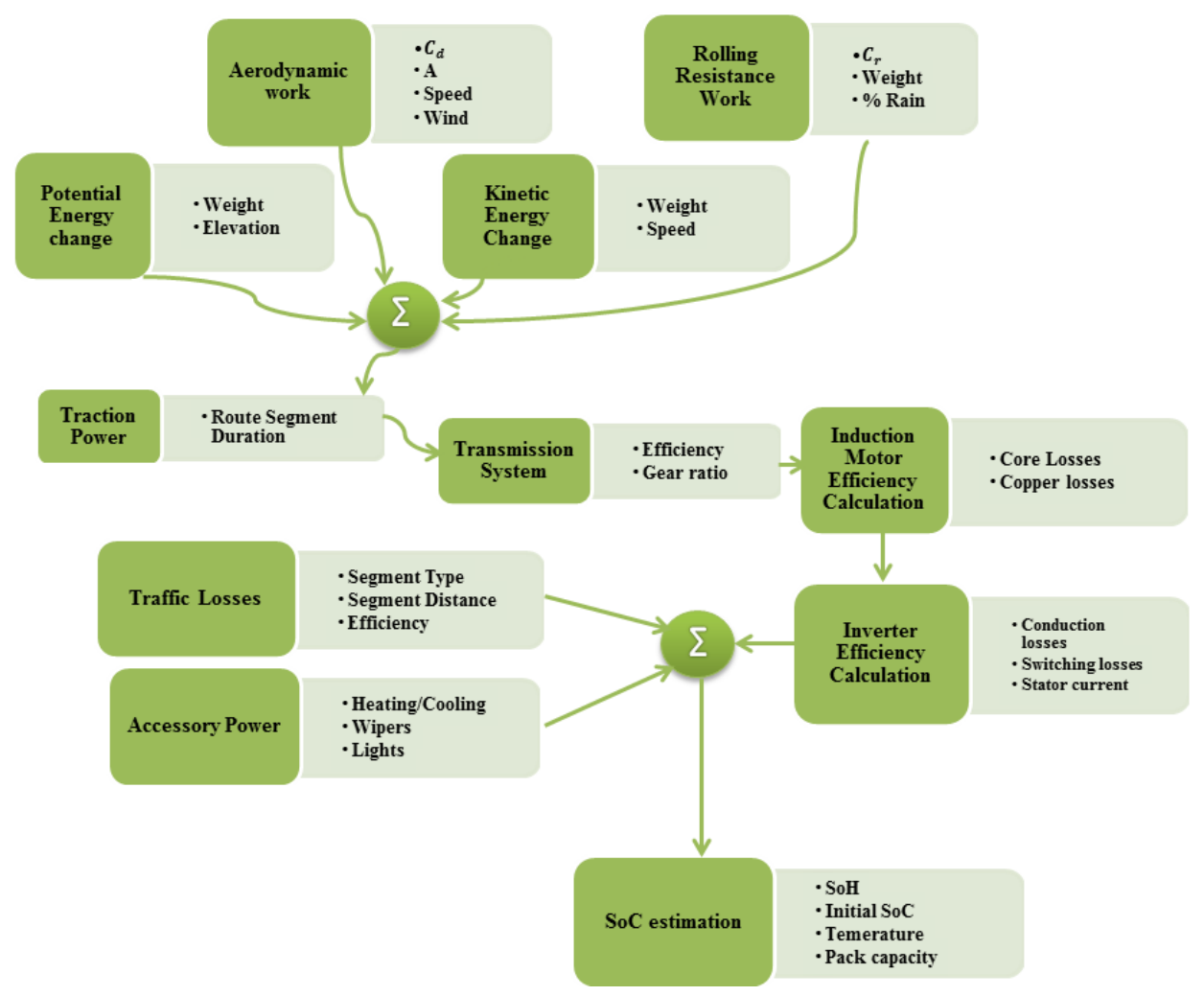

Fig. 2. Overview of the whole procedure for range estimation

Fig. 2 shows that to calculate the required energy from the battery, the traction power for a given route needs to be calculated first and it is the sum of the vehicle potential and kinetic energy change, the aerodynamic, and the rolling resistance work. Each of these components is highly dependent on a variety of environmental and behavioural factors. From the traction power, the EV motor output power can be calculated based on the transmission gear ratio and efficiency. Finally the required battery current required by the EV is dependent on the drive system efficiencies, the traffic conditions and the accessory power losses. 
The SoC of the EV battery and the vehicle range to destination at each instant of time can be estimated from the battery current required by the EV, the battery voltage, the initial SoC, battery temperature, capacity and efficiency. The proposed SoC estimation takes into account the changes in environmental and the driver's behavioural factors and the dynamic changes of the losses in the EV components.

\section{Traction power estimation considering dynamically varying environmental factors}

The wheel traction power, $P_{t}(W)$, can be calculated based on the inertial and external forces acting on $\mathrm{EV}[1,4,7]$ :

$$
P_{t}=\frac{\Delta E_{p}+\Delta E_{k}+W_{\text {aero }}+W_{\text {rolling }}}{t_{\text {segment }}}
$$

Where:

- $\Delta E_{p}$ is the potential energy change $(J)$

- $\Delta E_{k}$ is the kinetic energy change $(J)$

- $\quad W_{\text {aero }}$ is the aerodynamic work $(J)$

- $\quad W_{\text {rolling }}$ is the rolling resistance work $(J)$

- $\quad t_{\text {segment }}$ is the route segment duration $(s)$

\subsection{Driving route}

To evaluate the traction power, the specific driving route must first be selected and is presented as a series of points (route segment). The parameters for each route point such as latitude, longitude, speed limit and elevation can be extracted from Google Map, from which the length between two points can be calculated.

\subsection{Vehicle Speed and Time Duration for Each Segment}

In this paper, the speed of the vehicle is calculated based on the speed limit for each segment and the assumed nominal acceleration. This assumption is chosen based on the driver's behaviour for acceleration rate and the capability of the EV for acceleration. The predicted speed at the end of each route segment can be calculated by considering constant acceleration for that specific route segment using (2).

$$
v_{E V}=\sqrt{v_{0}^{2}+2 a L_{\text {segment }}}
$$

Where: 
- $\quad v_{E V}$ is the predicted vehicle speed at the end of the route segment $(\mathrm{m} / \mathrm{s})$

- $v_{0}$ is the vehicle speed at the start of the route segment $(\mathrm{m} / \mathrm{s})$

- $\quad a$ is the nominal acceleration $\left(\mathrm{m} / \mathrm{s}^{2}\right)$

- $\quad L_{\text {segment }}$ is the length of the route segment $(m)$

Since the length of each route segments from Google Map is usually quite small, using constant acceleration for each route segment based on the capability of the EV and driver's behaviour as well as the speed limitation produces negligible error. The segment time calculation is determined by dividing the segment length over the predicted EV speed up to the speed limit for each segment.

\subsection{Potential Energy Change}

For each route segment, the change in elevation can be calculated from the latitudes, longitudes and elevations for the starting and ending points of each segment. The potential energy change due to this change of elevation can be calculated by (3).

$$
\Delta E_{p}=m g\left(h_{i+1}-h_{i}\right)
$$

Where:

- $\quad m$ is the EV mass $(\mathrm{kg})$

- $g$ is the acceleration due to gravity $\left(\mathrm{m} / \mathrm{s}^{2}\right)$

- $\quad h_{i}$ is the elevation of point $i$ on the route $(m)$

The traction power due to gravity, $P_{\text {gravity }}(W)$, can be calculated using (4). The positive and negative values denote uphill and downhill road respectively.

$$
P_{\text {gravity }}=\frac{\Delta E_{p}}{t_{\text {segment }}}
$$

\subsection{Kinetic Energy Change}

The kinetic energy for a specific segment can be calculated from the speed variation in the segment as follows:

$$
\Delta E_{k}=0.5 m\left(v_{E V}^{2}-v_{0}^{2}\right)
$$

The traction power due to acceleration/deceleration, $P_{k}(W)$, can be calculated using (6).

$$
P_{k}=\frac{\Delta E_{k}}{t_{\text {segment }}}
$$

\subsection{Aerodynamic work}


The aerodynamic shape of the EV directly affects the power due to drag. In addition, wind speed and the direction of the wind are the crucial factors for calculating the actual speed due to drag. For precise calculation, the relative speed i.e. the effects of the wind speed and wind direction on vehicle speed need to be taken into account. The force due to drag, $F_{\text {aero }}(N)$, and the relative speed, $v_{r e l}(\mathrm{~m} / \mathrm{s})$, can be calculated as follows:

$$
\begin{gathered}
F_{\text {aero }}=\frac{1}{2} \rho_{\text {air }} C_{d} A v_{\text {rel }}^{2} \\
v_{\text {rel }}=v_{E V}+\left(w_{s} \times \cos \left(d i r_{E V}-d i r_{\text {wind }}\right)\right)
\end{gathered}
$$

Where:

- $\quad \rho_{\text {air }}$ is the air density $\left(\mathrm{kg} / \mathrm{m}^{3}\right)$

- $\quad C_{d}$ is the aerodynamic drag coefficient

- $\quad A$ is the vehicle frontal area $\left(m^{2}\right)$

- $\quad w_{s}$ is the wind speed $(\mathrm{m} / \mathrm{s})$

- $\quad \operatorname{dir}_{E V}$ is the heading of the EV (degree)

- $d i r_{\text {wind }}$ is the wind direction coming toward the EV (degree)

Based on the calculated time segment, for each segment the power due to drag, $P_{\text {aero }}(W)$, can be calculated using (9).

$$
P_{\text {aero }}=\frac{F_{\text {aero }} \times L_{\text {segment }}}{t_{\text {segment }}}
$$

To consider the direction of the EV, in each segment the course of the vehicle is considered. These calculations are not usually considered in reported papers.

\subsection{Rolling Resistance work}

The rolling resistance power can be calculated based on the rolling resistance coefficient of the tyres, which can be affected by three main parameters: 1) tyre pressure 2) road surface characteristics 3) tread conditions [4]. The tyre pressure is assumed to be constant along the route and the tread condition is negligible. However, the road surface characteristics have the most impact on the rolling resistance. It has been discovered that the rolling resistance will increase by up to $10 \%$ in comparison with the same road on a dry condition [1]. The rolling resistance work, $P_{\text {rolling }}(W)$, considering road surface characteristics can be calculated using (10) to (13).

$$
F_{\text {rolling }}=C_{r-\text { effective }} m g \cos \alpha
$$




$$
\begin{gathered}
C_{r-\text { effective }}=C_{r-d r y}+\left(\operatorname{prob}_{\text {rain }} \times\left(C_{r-\text { wet }}-C_{r-d r y}\right)\right) \\
W_{\text {rolling }}=F_{\text {rolling }} L_{\text {segment }} \\
P_{\text {rolling }}=\frac{W_{\text {rolling }}}{t_{\text {segment }}}
\end{gathered}
$$

Where:

- $\quad F_{\text {rolling }}$ is the rolling resistance force $(N)$

- $\quad C_{r \text {-effective }}$ is the effective rolling resistance coefficient

- $\quad \alpha$ is the slope angle $(100 \sin \alpha=\%$ slope $)$

- $\quad C_{r-d r y}$ is the dry value for $C_{r}$

- prob $_{\text {rain }}$ is the probability of rain $(\%)$

- $\quad C_{r \text {-wet }}$ is the wet value for $C_{r}$

In (11), the $C_{r-e f f e c t i v e}$ is calculated from $C_{r-d r y}$ plus the probability of rain times the difference between $C_{r \text {-wet }}$ and $C_{r-d r y}$ (which is assumed to be 10\%). The probability of rain for each segment can be derived from the weather forecast websites. In this way, there is a continuum of change in effective rolling resistance coefficient value of $C_{r \text {-effective }}$.

\section{Drive system Estimation}

Once the traction power is calculated by the addition of the vehicle potential and kinetic energy change, the aerodynamic, and the rolling resistance work, the traction power needs to be converted to the output power of the motor by using the transmission system gear ratio. The gear ratio, $G$, can be calculated using (14) $[6,8]$ :

$$
G=\frac{n_{s \max }}{v_{E V \max }} \times \frac{2 \pi}{60} \times \frac{r_{w}}{1.1}
$$

Where:

- $\quad n_{\text {smax }}$ is the maximum motor speed (rpm)

- $\quad v_{\text {EVmax }}$ is the maximum vehicle speed $(\mathrm{m} / \mathrm{s})$

- $\quad r_{w}$ is the wheel radius $(m)$

The traction torque, $T_{t}(\mathrm{Nm})$, and the load torque, $T_{L}(\mathrm{Nm})$, of the motor (see Fig. 1) can be calculated as follows: 


$$
\begin{gathered}
T_{t}=\frac{P_{t} \times r_{w}}{v_{E V}} \\
T_{L}=\left\{\begin{array}{l}
\frac{T_{t}}{\eta_{T S} \times G}, P_{t} \geq 0 \\
\frac{\eta_{T S} \times T_{t}}{G}, P_{t}<0
\end{array}\right.
\end{gathered}
$$

\section{Where:}

- $\quad \eta_{T S}$ is the transmission system efficiency

Due to the limited information on the transmission system for the actual EV used for the validation in this paper, the efficiency of the transmission system is considered to be $95 \%$ based on the information provided by [6].

The vehicle speed, $W_{t}(\mathrm{rad} / \mathrm{s})$, and the shaft speed, $W_{L}(\mathrm{rad} / \mathrm{s})$, of EV can be calculated using (17) and (18).

$$
\begin{gathered}
W_{t}=\frac{v_{E V}}{r_{w}} \\
W_{L}=\frac{G \times v_{E V}}{r_{w}}
\end{gathered}
$$

The electromagnetic torque at point $i+1$ on the road, $T_{\text {electromagnetic }, i+1}(\mathrm{Nm})$, based on the torque load, shaft speed and the nominal parameters of the motor can be calculated using (19).

$$
T_{\text {electromagnetic }, i+1}=J \frac{\left(W_{L, i+1}-W_{L, i}\right)}{t_{\text {segment }}}+B \times W_{L, i+1}+T_{L, i+1}
$$

Where:

- $\quad J$ is the moment of inertia $\left(k g \cdot \mathrm{m}^{2}\right)$

- $W_{L, i+1}$ is the shaft speed at $(i+1)^{t h}$ point along a route $(\mathrm{rad} / \mathrm{s})$

- $\quad B$ is the damping coefficient $\left(k g . \mathrm{m}^{2} / \mathrm{s}\right)$

- $\quad T_{L, i+1}$ is the torque load at $(i+1)^{t h}$ point along a route $(\mathrm{Nm})$

The output power of the motor at point $i+1$ on the road, $P_{\text {electromagnetic }, i+1}(W)$, is the electromagnetic power as shown in (20).

$$
P_{\text {electromagnetic }, i+1}=T_{\text {electromagnetic }, i+1} \times W_{L, i+1}
$$

\subsection{Modelling Induction Motor Losses}


The losses in all induction motors are mainly due to stator copper loss, rotor copper loss, core loss, stray loss and friction losses $[9,10,11]$. In [9] especially for efficiency computations of induction motors, only copper and core losses are modelled, because they are considered to be the main electrical losses in the induction motor.

Since the copper loss is a function of rotor and stator losses and core loss is proportional to the air gap voltage, the rated losses in induction motor can be calculated for a specific set of steady state parameters of the motor $[12,13]$. At any instant of time, the motor can operate in two regions: (i) constant torque region, when the motor is operating below its rated speed, and (ii) constant power region, when the motor is operating above its rated speed.

4.1.1 Constant Torque Region: By controlling $V / f$, the flux in the three phase induction motor can be kept constant in the air gap; as long as the speed of the motor is below the rated speed of the motor. Once the speed is above the rated speed, the flux will not be constant any more.

In this constant toque region, the motor can produce torque up to its rated value and the motor experiences constant flux. The current can be assumed to be proportional to the output torque (assuming that the power factor is constant), and the instantaneous copper loss at different points of driving cycle, $P_{c u}$ achieved $(W)$, can be calculated as follows:

$$
\begin{gathered}
P_{\text {cu-achieved }}=\left[\frac{P_{\text {electromagnetic }}}{P_{\max }} \frac{W_{\text {rated }}}{W_{L}}\right]^{2} P_{\text {cu-rated }} \\
P_{\text {cu-rated }}=3 R_{s} I_{s}^{2}+3 R_{r} I_{r}^{2}
\end{gathered}
$$

Where:

- $\quad P_{\text {electromagnetic }}$ is the electromagnetic power $(W)$

- $\quad P_{\text {max }}$ is the maximum motor power $(W)$

- $\quad W_{\text {rated }}$ is the rated motor speed $(\mathrm{rad} / \mathrm{s})$

- $\quad P_{\text {cu-rated }}$ is the rated copper loss $(W)$

- $\quad R_{S}$ is the rated stator resistance $(\Omega)$

- $\quad I_{s}$ is the rated stator current $(A)$

- $\quad R_{r}$ is the rated rotor resistance $(\Omega)$

- $\quad I_{r}$ is the rated rotor current $(A)$

In the constant torque region, the flux to a large extent can be assumed constant. Based on Steinmetz expression [14], the eddy current loss is proportional to the square of the flux and the frequency but the flux in this region is constant. Thus, the eddy current loss is only proportional to the square of the 
frequency. The same situation can be applied for the hysteresis loss calculation. Since the frequency is proportional to the motor speed, the total core loss, $P_{\text {fe-achieved }}(W)$, in this region can be expressed as follows:

$$
\begin{gathered}
P_{f e-\text { achieved }}=\left[\frac{W_{L}}{W_{\text {rated }}}\right]^{2} P_{e-\text { rated }}+\left[\frac{W_{L}}{W_{\text {ratred }}}\right] P_{h-\text { rated }} \\
P_{f e-\text { rated }}=P_{e-\text { rated }}+P_{h-\text { rated }}
\end{gathered}
$$

Where:

- $\quad P_{f e-r a t e d}$ is the rated core loss $(W)$

- $\quad P_{\text {e-rated }}$ is the rated eddy loss $(W)$

- $\quad P_{h \text {-rated }}$ is the rated hysteresis loss $(W)$

4.1.2 Constant Power Region: In this region, the motor speed is operating above its rated speed and the power and the voltage of the motor are above their rated values. The stator frequency of the motor is also increased to the value above its rated value causing $V / f$ to be reduced and the flux declines based on the instantaneous operating speed. Using this assumption the copper loss in the constant power region becomes:

$$
P_{\text {cu-achieved }}=\left[\frac{P_{\text {electromagnetic }}}{P_{\max }}\right]^{2} P_{c u-\text { rated }}
$$

For core loss calculation, in the stator or rotor core of the motor, the flux varies inversely with respect to the frequency, so flux $\times$ frequency will be constant. Thus, the eddy current loss in this region is kept constant and the hysteresis loss can be shown to be proportional to the power of 1.6 of the frequency and is expressed as below [10]:

\subsection{Modelling Inverter Losses}

$$
P_{\text {fe-achieved }}=P_{e-\text { rated }}+\left[\frac{W_{L}}{W_{\text {ratred }}}\right]^{1.6} P_{h-\text { rated }}
$$

A standard six-switch three-phase bridge inverter is considered for the calculation of the losses and efficiency of the power converter of the electric traction motor drive.

The major losses for each switching component are the conduction losses and the switching losses $[9,15]$. The conduction losses are load dependent and will vary according to the stator current of the motor. The switching loss is constant for the diode and the output power has no effect on the diode switching losses. The switching losses in the switch, however, are load-dependent with respect to the voltage and current. 
4.2.1 Loss modelling in the switch: The conduction, $P_{\text {cond, } Q}(W)$, and switching, $P_{s w, Q}(W)$, losses of the MOSFET or IGBT switch are elaborated below $[16,17,18]$ :

$$
\begin{gathered}
P_{Q}=P_{c o n d, Q}+P_{s w, Q} \\
P_{c o n d, Q}=I_{Q, a v e} V_{C E}+I_{Q, r m s}^{2} R_{C E, o n}
\end{gathered}
$$

Where:

- $\quad P_{Q}$ is the total switching loss $(W)$

- $\quad I_{Q \text {,ave }}$ is the switch average current $(A)$

- $I_{Q, r m s}$ is the switch RMS current $(A)$

- $\quad V_{C E}$ is the constant voltage drop of the switch $(V)$

- $R_{C E, \text { on }}$ is the switch on-resistance $(\Omega)$

It is assumed that the inverter controller is controlled using the pulse-width modulation type. Hence, for the three phase inverter, the conduction losses can be calculated as follows $[16,17,18]$ :

$$
P_{\text {cond }, Q}=\left(\frac{1}{8}+\frac{M}{3 \pi} \cos (\theta)\right) R_{C E, o n} I_{Q}^{2}+\left(\frac{1}{2 \pi}+\frac{M}{8} \cos (\theta)\right) I_{Q} V_{C E}
$$

Where:

- $\quad M$ is the modulation index

- $\operatorname{Cos}(\theta)$ is the power factor

- $\quad I_{Q}$ is the switch current $(A)$

The switching losses in the switch are dependent on the switch voltage, $V_{Q}(V)$, the stator current of the motor, the turn-on rise-time, $t_{o n, s w}(s)$, and the turn-off fall-time, $t_{\text {off,sw }}(s)$, of the switch and the switching frequency $f_{s w}(\mathrm{~Hz})$. The average switching losses in the switch can be calculated as follows $[15,18]$ :

$$
P_{s w, Q}=\frac{V_{Q} I_{Q}}{2} f_{s w}\left(t_{o n, s w}+t_{\text {off }, s w}\right)
$$

4.2.2 Loss modelling in the Diode: The duty cycle of the anti-parallel diode is different from the duty cycle of the switch since when the switch is off in a lagging circuit, the flow of current in diode is positive until the current reaches zero and consequently the diode will be still on even when the switch is off. The conduction loss in diode, $P_{\text {cond,Diode }}(W)$, can be calculated using $(31)[9,16,18]$.

$$
P_{\text {cond,Diode }}=\left(\frac{1}{8}-\frac{M}{3 \pi} \cos (\theta)\right) R_{\text {Diode,on }} I_{\text {Diode }}^{2}+\left(\frac{1}{2 \pi}-\frac{M}{8} \cos (\theta)\right) I_{\text {Diode }} V_{\text {Diode }}
$$

Where:

- $\quad R_{\text {Diode, on }}$ is the diode on-resistance $(\Omega)$ 
- $\quad I_{\text {Diode }}$ is the diode current $(A)$

- $\quad V_{\text {Diode }}$ is the diode Voltage $(V)$

Besides the conduction loss, the prominent component in diode switching losses is the reverse recovery losses while the other components in diode switching losses are quiet small and negligible [18]. The switching losses of the anti-parallel diode, $P_{s w, \text { Diode }}(W)$, can be calculated using (32) [9].

$$
P_{s w, \text { Diode }}=\frac{f_{s w} V_{R}}{2 S}\left(\frac{d I_{F}}{d t}\right)\left(\frac{S t_{r r}}{S+1}\right)
$$

Where:

- $\quad V_{R}$ is the RMS reverse voltage $(V)$

- $\quad S$ is the snap factor

- $\quad d I_{f} / d t$ is the rate of fall forward current $(A / s)$

- $\quad t_{r r}$ is the reverse recovery time $(s)$

Finally, the total inverter losses can be calculated by multiplying the total losses in one switch and one diode by a factor of 6 in a typical three-phase inverter drive. There are still other losses that need to be considered to evaluate the total energy required by the battery, such as traffic conditions and energy drawn for auxiliary loads.

\section{Traffic Condition}

The energy used to accelerate the EV cannot be fully recaptured during deceleration as the motor efficiency is not constant as explained in section 4. In this paper, the energy loss during acceleration and deceleration at each stop is not only dependent on the vehicle speed but also is directly dependent on the efficiency of each segment of the EV and can be calculated as follows:

$$
E_{\text {Traffic }}=\frac{m v_{E V}^{2}}{2}\left(\frac{1}{\eta_{\text {motor }}}-\eta_{\text {regen }}\right)
$$

Where:

- $\quad E_{\text {Traffic }}$ is the traffic losses $(W)$

- $\quad \eta_{\text {motor }}$ is the motor efficiency $(\%)$

- $\quad \eta_{\text {regen }}$ is the regenerative braking efficiency (\%)

In order to add the effect of the traffic conditions, four traffic conditions are considered in this paper as shown in Table 1. From Table 1, for each route segment, 'segment call sign' was allocated based on the number of stops in each kilometre. For the particular kilometre (with specific number of segments), the average speed of the EV and the average efficiency of the drive system in motor mode and regenerative mode were calculated. Therefore, the energy loss due to the traffic for the particular kilometre for each 
stop can be obtained using (33) and finally, the total energy loss can be considered to be equal to the number of stops in the particular kilometre (defined by the 'segment call sign') multiplied by $E_{\text {Traffic }}$.

Table 1 Traffic Condition along a route

Four traffic conditions $\quad$ Stops $/ \mathrm{km} \quad$ Segment call sign

\begin{tabular}{lll} 
Highway without stops & 0 & 1 \\
Light traffic condition & 1 & 2 \\
Moderate traffic condition & 3 & 3 \\
Heavy traffic & 5 & 4 \\
\hline
\end{tabular}

The traffic power, $P_{\text {Traffic }}(W)$, for each segment can be calculated by dividing the traffic energy for each segment over the segment time as follows:

$$
P_{\text {Traffic }}=\frac{E_{\text {Traffic }}}{t_{\text {segment }}}
$$

\section{Auxiliary loads}

In order to have an accurate SoC and range estimation model, it is essential to consider a number of significant loads which are normally present when driving a vehicle such as the air conditioning system, windscreen wipers and lighting.

\subsection{Air conditioning power}

The cabin temperature and the weather condition are the prominent factors for modelling the air conditioning power. The position and the colour of the side windows, sunlight exposure are the other factors that can have effects on the air conditioning power are not considered in this paper. The necessary power for air conditioning power, $P_{a c}(W)$, can be calculated using (35).

$$
P_{a c}=\left\{\begin{array}{l}
0, \text { off } \\
P_{\text {heat }}\left(T_{\text {cabin }}-T_{\text {air }}\right), \text { heating } \\
P_{\text {cool }}\left(T_{\text {air }}-T_{\text {cabin }}\right), \text { cooling }
\end{array}\right.
$$

Where:

- $\quad P_{\text {heat }}$ is the heating power per degree $\left(W /{ }^{\circ} C\right)$

- $\quad P_{\text {cool }}$ is the cooling power per degree $\left(W /{ }^{\circ} \mathrm{C}\right)$

- $T_{\text {cabin }}$ is the cabin temperature set point $\left({ }^{\circ} \mathrm{C}\right)$

- $\quad T_{\text {air }}$ is the ambient temperature $\left({ }^{\circ} \mathrm{C}\right)$

\subsection{Windscreen wipers:}


The probability of the rain has a main effect on the proportion of the time that the wipers operate. Hence, it is necessary to calculate the required power for the operation of windscreen wipers, $P_{\text {wiping }}(W)$, based on the probability of the rain as follows:

$$
P_{\text {wiping }}=\operatorname{prob}_{\text {rain }} P_{\text {wiper }}
$$

Where:

- $\quad P_{\text {wiper }}$ is the power required to run the windscreen wipers continuously $(W)$

\subsection{Lighting:}

In this paper it is assumed that the lighting power is necessary during the night. Hence, the lighting power, $P_{\text {lighting }}(W)$, can be expressed by (37).

$$
P_{\text {lighting }}=\left\{\begin{array}{l}
0, \text { day } \\
P_{\text {light }}, \text { night }
\end{array}\right.
$$

Where:

- $\quad P_{\text {light }}$ is the rated power required to run the vehicle light $(W)$

\section{Total estimated power and current drawn from the battery}

Finally, the total battery power, $P_{\text {Battery }}(W)$, and the required battery current, $I_{\text {Battery }}(A)$, at each segment can be calculated using (38) and (39).

$$
\begin{gathered}
P_{\text {Battery }}=P_{T B}+P_{\text {Traffic }}+P_{\text {AC }}+P_{\text {lighting }}+P_{\text {wiping }} \\
I_{\text {Battery }}=\frac{P_{\text {Battery }}}{V_{\text {Battery }}}
\end{gathered}
$$

Where:

- $\quad P_{T B}$ is the traction power seen from the battery side $(W)$

- $\quad V_{\text {Battery }}$ is the battery pack voltage $(V)$

\section{SoC estimation}

In this paper, for the SoC estimation, a developed SoC algorithm using low-cost microcontrollers proposed in [19] is used. The SoC for each segment can be calculated based on operating parameters such as battery current during charge and discharge, temperature, the open-circuit voltage (OCV) of the cell, etc. In addition, the State of Health $(\mathrm{SoH})$ of the battery (the effective battery capacity when fully charged over 
the rated battery capacity) needs to be considered in SoC estimation because the effective battery capacity will be changed by many factors. In this paper, three correction factors are considered for the corrected battery capacity and SoC estimations as follows:

$$
\operatorname{SoC}(t)=\operatorname{SoC}\left(t_{0}\right)-\frac{\int_{T} I_{\text {Battery }} d t}{Q_{A}}
$$

For a specific cycle, $Q_{A}$ is the latest value of the cumulative moving average of the expected total battery capacity $(A h)$ and can be calculated as follows [19]:

$$
Q_{A}=\left(Q_{C}+i Q_{A i-1}\right) /(i+1)
$$

Where:

- $\quad \operatorname{SoC}\left(t_{0}\right)$ is the initial SoC $(\%)$

- $\quad i$ is the index for each segment

- $\quad Q_{A i-1}$ is the prior value of $Q_{A}(A h)$

- $Q_{C}$ is the corrected battery capacity (Ah)

By taking into account the current and temperature based on the last reading, $Q_{C}$ can be calculated using (42) [19].

$$
Q_{C}=\lambda_{C} \lambda_{T} \lambda_{\text {SoH }} Q_{R}
$$

Where:

- $\lambda_{C}$ is the correction factors due to charge or discharge rate

- $\quad \lambda_{T}$ is the correction factor due to the cell temperature

- $\lambda_{\text {SoH }}$ is the current $\mathrm{SoH}$ of the cell

- $\quad Q_{R}$ is the rated capacity of the cell $(A h)$

In (42), $\lambda_{C}$ and $\lambda_{T}$ values were obtained from experimental tests and the results are then collected in a look-up table. The procedures of the methods for determining the $\lambda_{C}$ and $\lambda_{T}$ are fully explained in [19]. During the experimental tests, the measured values of $\lambda_{C}$ and $\lambda_{T}$ are obtained by interpolation from the look-up table for different operating temperature, charge and discharge rate. The corrected battery capacity value will be updated by using the lambda correction factors obtained from the interpolation. Meanwhile, the initial SoC and the pack SoH needs to be provided by the vehicle's BMS at the start of the experiment. The lambda correction factors were in fact not constant, and in this paper the look-up table method provided in $[1,19]$ was used. 


\section{Validation of the Proposed Range Estimation Algorithm}

To validate the accuracy of the proposed algorithm, an Electron Blade EV was driven along a route, which includes both urban and freeway driving conditions with significant change in elevation from $18 \mathrm{~m}$ to $449 \mathrm{~m}$ shown in Fig. 3. The trip originated at the UoW Innovation Campus at 9:00AM local time and the air temperature was $16^{\circ} \mathrm{C}$ at that time. From wunderground website [20], the observed wind speed was $11 \mathrm{~km} / \mathrm{hr}$ and was from the southwest with scattered showers along a route. From the website, it is possible to extract the information of wind speed, direction and the probability of the rain for each latitude and longitude along a route on that particular day. Therefore, different values for wind speed, wind direction and the probability of the rain for each segment have been used.

The journey starts by driving north with a speed limit between $60 \mathrm{~km} / \mathrm{h}$ and $70 \mathrm{~km} / \mathrm{h}$ (urban and suburban areas) with lots of roundabouts and stop lights. At the suburb of Bulli, the route turns westward to climb Bulli pass to the top of Mount Ousley. In order to consider the effects of the regenerative braking system, the route then joins the M1 freeway at $100 \mathrm{~km} / \mathrm{h}$ for a climb to a peak of $445 \mathrm{~m}$ above sea level and then descending back to $34 \mathrm{~m}$ elevation at the exit to Wollongong. Finally, the trip continues to the originating point with a total distance of $31.5 \mathrm{~km}$. The air conditioning system is also used to maintain the cabin temperature at $22^{\circ} \mathrm{C}$ at all time.

Sample EV dynamic parameters are shown in Table 2. Because of the data limitation on the tested EV used in this paper, typical values of $C_{r-d r y}$ and $C_{r \text {-wet }}$ for an automotive tyre are estimated [21]. The difference in predicted energy usage for roads with zero and $10 \%$ variation in rolling resistance have been estimated for one segment of the selected route to see if the difference in the predicted rolling resistance will make a significant difference in the predicted energy usage. The results demonstrated that the difference between dry surface condition and wet surface condition is only $0.867 \%$. Therefore, the difference in predicted rolling resistance will not make a significant difference in predicted energy usage. In addition, the drag coefficient obtained by the manufacturer data for the Electron Blade EV [22] and the frontal area measured during the validation process.

Table 2 EV parameters

\begin{tabular}{lcc}
\hline Parameter & Symbol & Value (Unit) \\
\hline Loaded vehicle weight & $m$ & $1340 \mathrm{~kg}$ \\
Rolling resistance coefficient (dry condition) & $C_{r-d r y}$ & 0.02 \\
Rolling resistance coefficient (wet condition) & $C_{r-w e t}$ & 0.022 \\
Acceleration due to gravity & $g$ & $9.8 \mathrm{~m} / \mathrm{s}^{2}$ \\
Air density & $\rho$ & $1.2754 \mathrm{~kg} / \mathrm{m}^{3}$ \\
Wheel radius & $r$ & $0.343 \mathrm{~m}$ \\
\hline
\end{tabular}




\begin{tabular}{lcc}
\hline Frontal area & $A$ & $2.5 m$ \\
Drag coefficient & $C_{d}$ & 0.33 \\
Gear ratio & $G$ & 4.091 \\
\hline
\end{tabular}

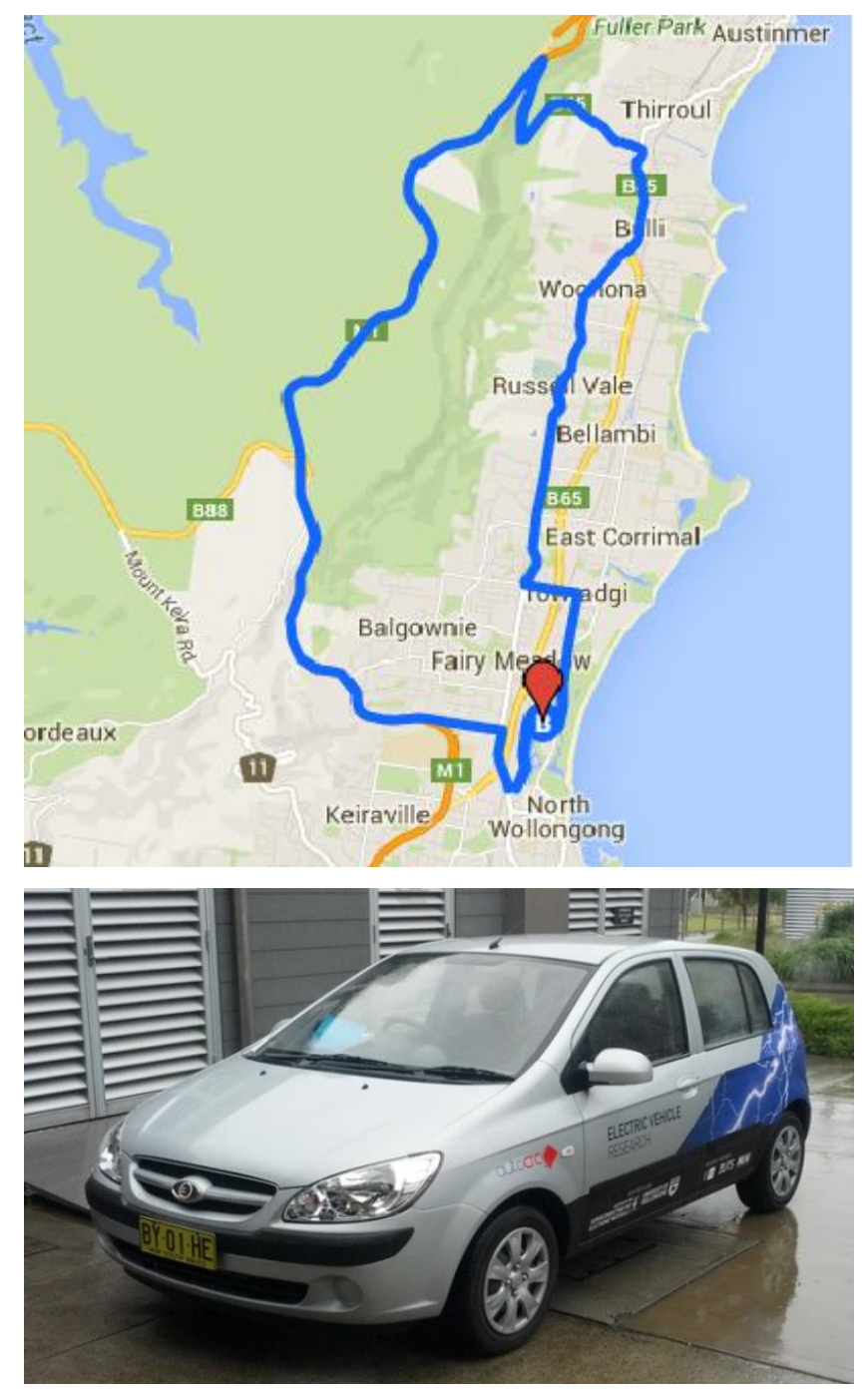

Fig. 3. Road map and converted Hyundai Getz (courtesy of the Institute for Superconducting and Electronic Materials (ISEM) at the $U o W)$

The measured battery current was obtained using an Orion Battery Management System (BMS) installed in the EV used while driven along a route. After the drive, the recorded battery current data was used as the input to the SoC estimation algorithm described in [19] to calculate the SoC of the battery along the route and at the destination point. This is expected to be a close representation of the actual SoC of the battery during this trip due to the validation of the SoC estimation algorithm as discussed in [19]. It has been referred as 'the measured SoC' because it is based on the measured battery current.

The difference between relative speed and vehicle speed is presented in Fig. 4. 


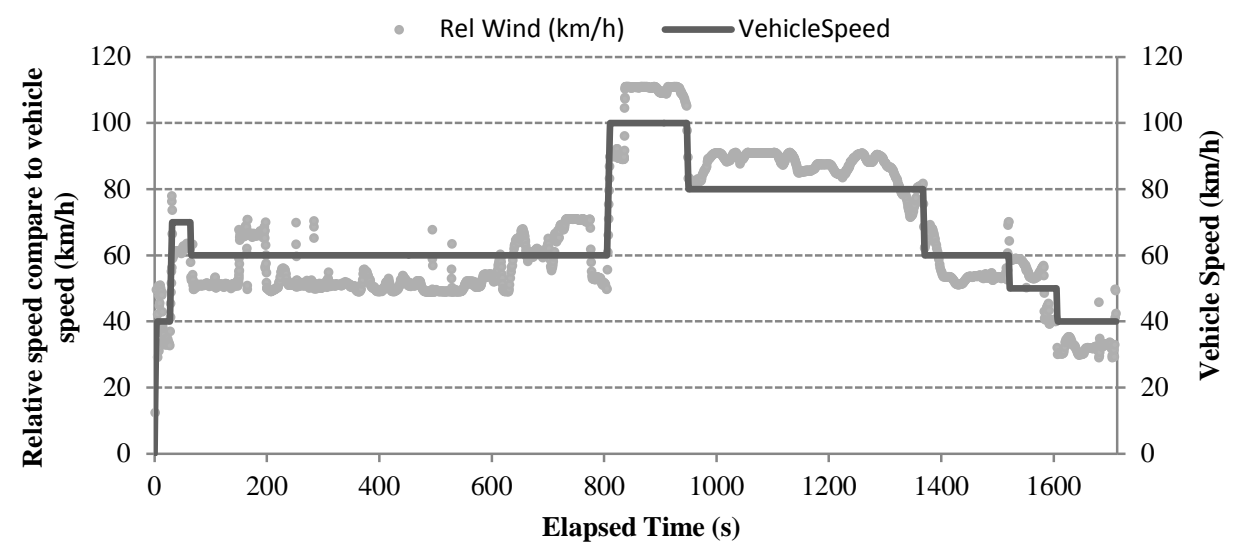

Fig. 4. Difference between relative speed and vehicle speed

Fig. 4 shows that sometimes the wind was blowing in the opposite direction of travel of a vehicle and therefore the relative speed was below the vehicle speed. Also sometimes, the headwind increased the relative speed and hence its acceleration power requirement. Therefore, the wind speed and wind direction can have a significant effect in the calculation of power due to acceleration.

The total traction power of the selected route with respect to the elevation profile is shown in Fig.5.

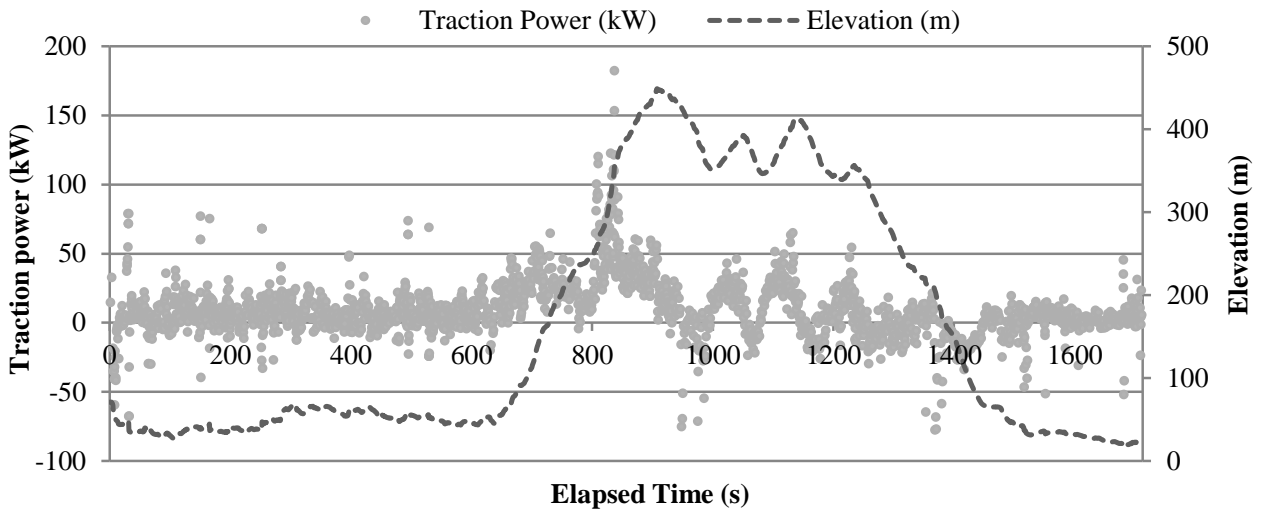

Fig. 5. Calculated traction power with a schematic view of change in elevation

Based on the calculated traction power along a route as shown in Fig. 5, the required energy with and without regenerative braking are $4.135 \mathrm{kWh}$ and $5.578 \mathrm{kWh}$ respectively in 1711 seconds. Based on the calculation of the rated losses for the $30 \mathrm{~kW}$ induction motor, the motor loss profile for the selected route for different speed in each segment is shown in Fig. 6(a). The loss calculations are done based on the motor equivalent circuit parameters which are shown in Table 3.

Table 3 Equivalent circuit parameters of a 30kW induction motor [23]

\begin{tabular}{lcc} 
Parameter & Symbol & Value (Unit) \\
\hline Rated power & $P_{\text {rated }}$ & $30(\mathrm{~kW})$ \\
Rated Frequency & $f_{\text {rated }}$ & $60(\mathrm{~Hz})$
\end{tabular}




\begin{tabular}{lcc}
\hline Number of poles & $P$ & $4(-)$ \\
Stator resistance & $R_{s}$ & $0.087(\Omega)$ \\
Rotor resistance & $R_{r}$ & $0.228(\Omega)$ \\
Stator inductance & $L_{1 s}$ & $0.0008(\mathrm{H})$ \\
Magnetizing inductance & $L_{m}$ & 0.0347 \\
Rotor inductance & $L_{1 r}$ & $0.0008(\mathrm{H})$ \\
\hline
\end{tabular}

The motor efficiency based on the calculated output power of the motor is shown in Fig. 6(b). It shows that the calculated efficiency in each segment is not constant, but varying. In some cases, the efficiency is equal to zero because the EV is in regenerative braking mode and the amount of power is very small compared to the constant losses in the motor (core losses). At these points there is no transfer of power from the battery side to the wheel. The average motor efficiency is found to be $87 \%$ which is close to the expected efficiency of the motor studied.

The power switching device used in this paper for the purpose of loss calculation was FQPF12N60CT 600V N-Channel MOSFET, and the anti-parallel power diode parameters were extracted from International Rectifier fast soft recovery diode 20ETF Quiet IR Series. The total MOSFET and diode losses based on the module selection in this paper are shown in Figs. 6(c) and 6(d) respectively.

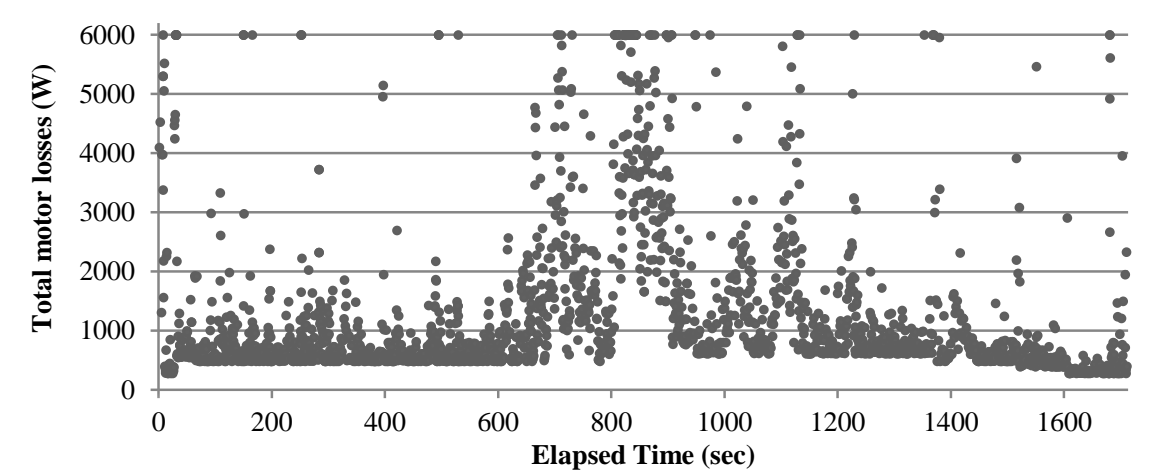

(a)

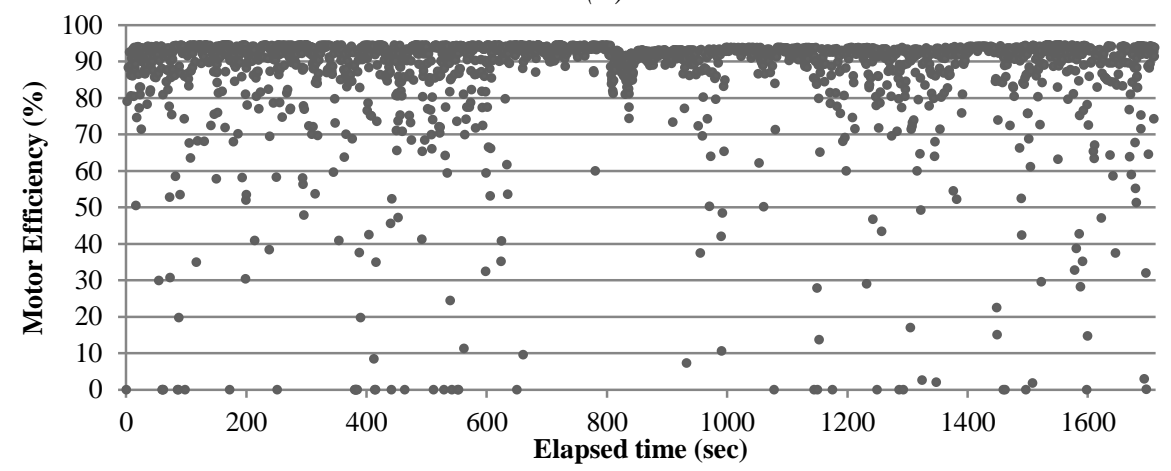

(b) 


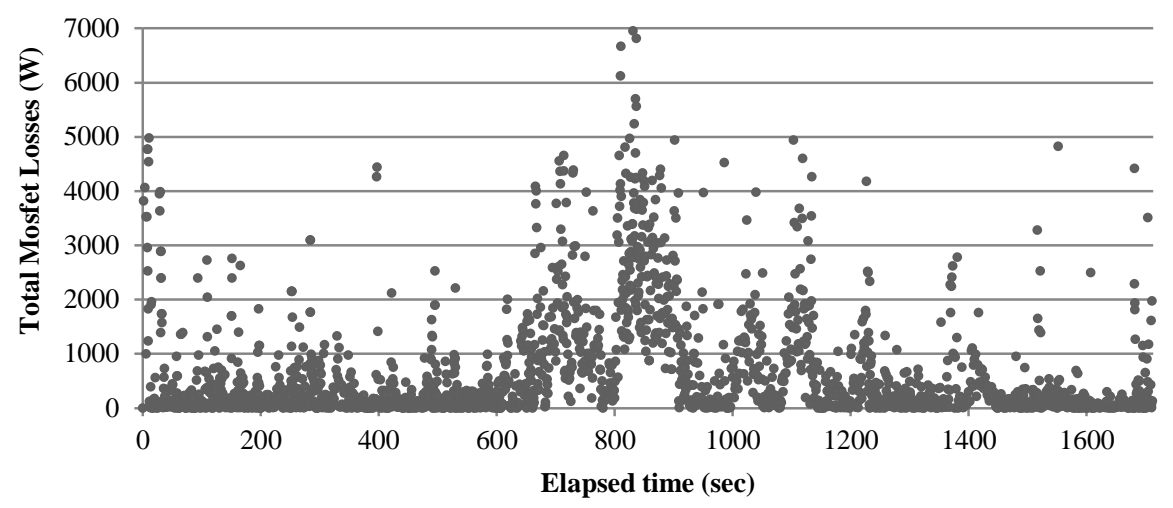

(c)

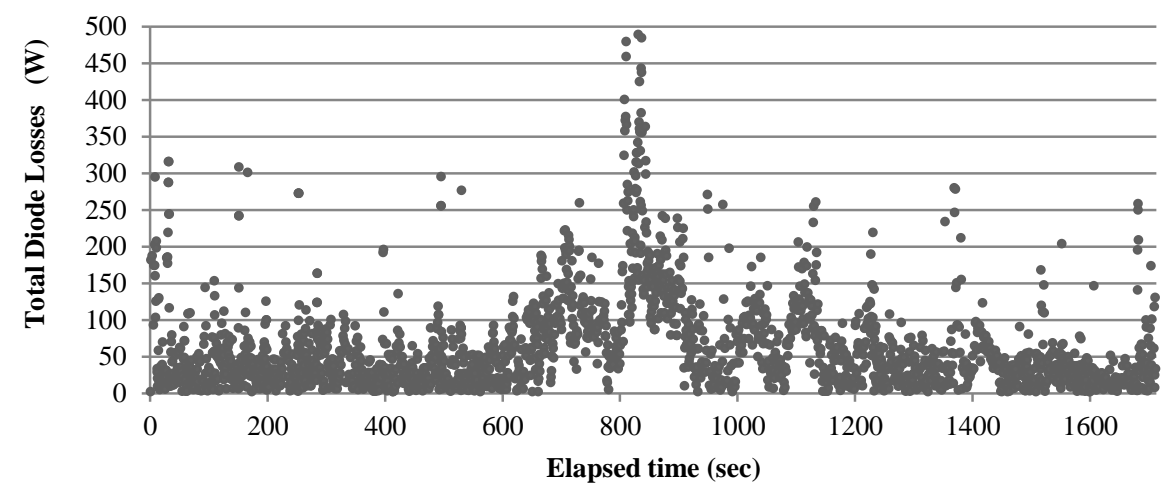

(d)

Fig. 6. Motor and inverter losses/efficiencies analysis:

(a) Total motor losses in motor

(b) Motor efficiency considering regenerative braking

(c) Total MOSFET losses

(d) Total diode losses

The average diode and MOSFET losses are $65 \mathrm{~W}$ and $1053 \mathrm{~W}$ respectively. The average efficiency for the inverter is $95 \%$, which is slightly higher than the reported efficiency of the inverter used in the EV. This is because the chosen inverter is a simple six-switch three-phase bridge inverter. The calculation, however, shows that if the actual inverter circuit is provided, the losses can be calculated more accurately than just assuming constant inverter efficiency commonly considered in range estimation calculations. The average system efficiency along the route is roughly $84 \%$, which is typical of the efficiency of the traction drive of an EV.

Fig. 7 shows the rate of change of SoC with the elevation profile along the selected route. During the acceleration the rate of change of SoC is negative and during the deceleration the rate of change of SoC is positive because of the regenerative braking mode. 


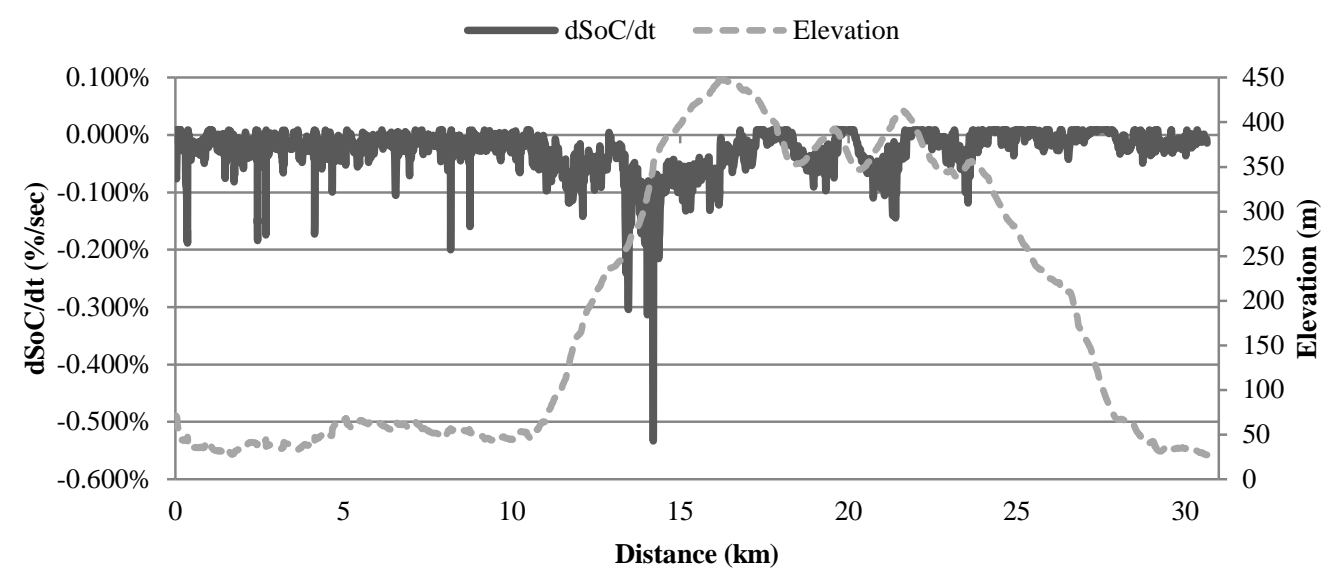

Fig. 7. Rate of change of SoC and Elevation vs. Distance

The comparison between the estimated SoC values considering constant efficiency proposed by [1], the estimated SoC values based on the calculated efficiency values along a selected route as proposed in this paper and the calculated SoC from the measured data is shown in Fig. 8.

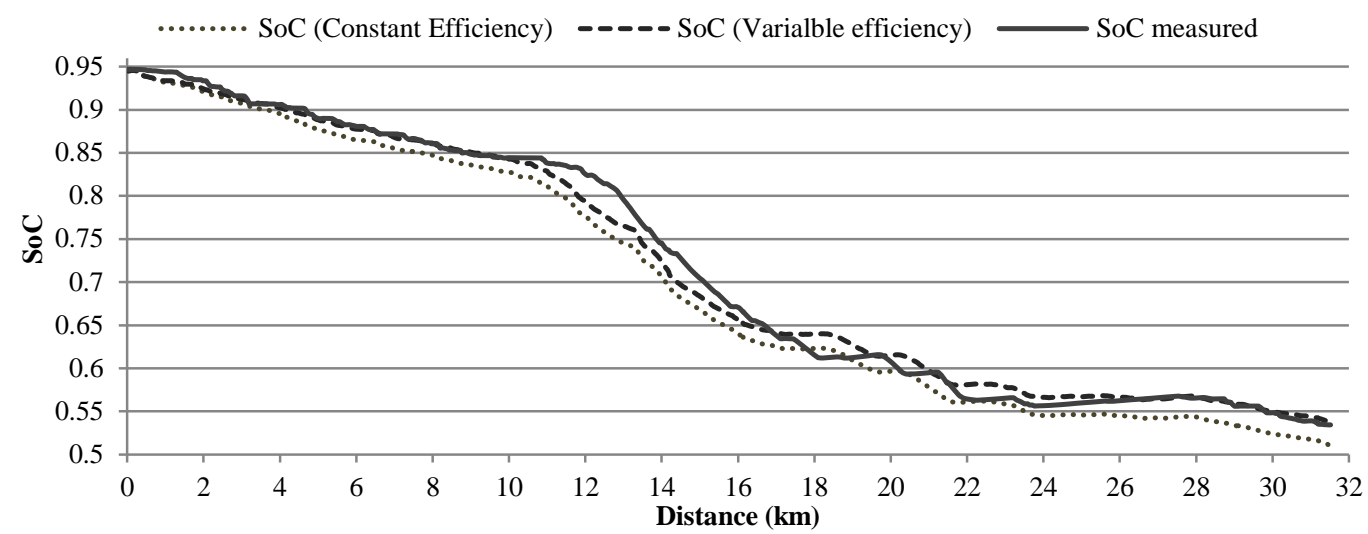

Fig. 8. SoC comparison along the selected route

It can be seen from Fig. 8 that the actual value for the SoC is approximately $53.4 \%$ at the destination. By calculating the efficiency of the traction system along the selected route as proposed in this paper, the estimate value for the SoC would be $53.7 \%$. The total error of the proposed model between the actual SoC at the destination and the predicted value is less than $0.5 \%$ for the validation drive as shown in Table 4 . The proposed method has performed better due to the added complexity of considering the varying efficiency of the traction drive of the EV, and the effect of varying wind speed and direction.

Table 4 Numerical results

Actual SoC at Destination $\quad 53.40 \%$




\section{Conclusion}

In this paper, an accurate $\mathrm{SoC}$ and range estimation model for EVs was described. The net battery energy usage was estimated, taking into account the time-domain losses and efficiency of the traction system along the specified route, and including environmental and behavioural factors. The traction system efficiency varied throughout the route, and was found to be $84 \%$ on average. The SoC calculation also took into account auxiliary loads such as windscreen wipers, air conditioning power, and lighting, and was found to be more accurate than previously reported methods, with only $0.5 \%$ difference between the estimated and measured value at the destination. Further work is being conducted to develop a real-time updating system using information obtained via a wireless internet connection from various sources such as Google Maps, meteorology sources, etc.

\section{Acknowledgments}

The authors thank the ISEM at the UoW for use of their EV and battery testing facility. This research is supported by the Australian Research Council and Optus, [LP130100811].

\section{References}

[1] Tannahill, V.R., Muttaqi, K.M., Sutanto, D.:'Driver alerting system using range estimation of electric vehicles in real time under dynamically varying environmental conditions', IET Electr. Syst. Transp., 2015, pp. 1-10

[2] Graser, A., Asamer, J., Dragaschnig, M.:'How to Reduce Range Anxiety?The Impact of Digital Elevation Model Quality on Energy Estimates for Electric Vehicles', GI Forum, 2014, pp. 165-174.

[3] Shankar, R., Marco, J.:'Method for estimating the energy consumption of electric vehicles and plug-in hybrid electric vehicles under real-world driving conditions', IET Intel. Transport Syst., 2013, 7(1), pp. 138-150

[4] Prins, R., Hurlbrink, R., Winslow, L.:'Electric vehicle energy usage modelling and measurement', IJME, 2013, 13(1), pp. $5-12$

[5] Vaz, W., Nandi, A.K.R., Landers, R.G., et al.:'Electric vehicle range prediction for constant speed trip using multiobjective optimization, J. Power Sources, 2015, 275, pp 435-446

[6] Schaltz, E.:'Electrical vehicle design and modeling' (INTECH Open Access Publisher, 2011)

[7] Wu, X., Freese, D., Cabrera, A., et al.:,'Electric vehicles' energy consumption measurement and estimation, Transp. Res. Part D, 2015, 34, pp 52-67

[8] Tabbache, B., Kheloui, A., Benbouzid, M.:'Design and control of the induction motor propulsion of an electric vehicle'. Proc. IEEE VPPC, Lille, France, Sep. 2010, pp. 1-6. 
[9] Williamson, S., Emadi, A., Rajashekara, K.:'Comprehensive Efficiency Modeling of Electric Traction Motor Drives for Hybrid Electric Vehicle Propulsion Applications', IEEE Trans. Veh. Technol., 2007, 56, pp. 1561-1572

[10] Rizzoni, G., Guzzella, L., Baumann, B.M.:'Unified modeling of hybrid electric vehicle drivetrains', IEEE/ASME Trans. Mechatron, 1999, 4, pp. 246-257

[11] Boldea, I., and Nasar, S. A.:'The induction machine handbook' (CRC press, 2010)

[12] El-Sharkawi, M. A.:'Fundamentals of Electric Drives, Brooks' (Cole Publishing Company, 2000)

[13] Cathey, J.:'Electric machines, analysis and design, applying MATLAB', (McGraw-Hill, 2001)

[14] Soulard, J., Meier, S., Chin, Y.:'Modelling of iron losses in permanent magnet motors with field-weakening capability'. Proc. NorPie, Sweden, 2002.

[15] Mohan, N., and Undeland, T.M.:'Power electronics:converters, applications, and design' ((John Willey\&Sons, 2001, 3rd edn.)

[16] Casanellas, F.:'Losses in PWM inverters using IGBTs', IEE P-Elec. Pow. Appl., 1994, 141, pp. $235-239$

[17] Dahono, P.A., Sato, Y., Kataoka, T.:'Analysis of conduction losses in inverters', IEE P-Elec. Pow. Appl., 1995,142, pp. $225-232$

[18] Istardi, D.:'Modeling and energy consumption determination of an electric go-kart'. M.Sc thesis, Chalmers University of Technology, Sweden, 2009

[19] Tannahill,V.R., Sutanto, D., Muttaqi, K.M., et al.:'Future vision for reduction of range anxiety by using an improved state of charge estimation algorithm for electric vehicle batteries implemented with low-cost microcontrollers, IET Electr. Syst. Transp., 2015, 5(1), pp 24-32

[20] 'WU (Weather Underground)', https://www.wunderground.com, Accessed March 2016.

[21] Gillespie, T.D.:'Fundamentals of vehicle dynamics' (SAE International, R-114, ISBN of 978-1-56091-199-9, 1992)

[22] '2008 Hyundai Getz Phase-II Hatchback all Versions Specifications and Performance Data', http://www.automobilecatalog.com/make/hyundai/click/getz_phase-ii_hatchback/2008.html, Accessed March 2016.

[23] Nayeem, H.S.M.,:'Hybrid electric vehicle powertrain:on-line parameter estimation of an induction motor drive and torque control of a PM BLDC starter-generator'. PhD thesis, University of Akron, US , 2008 